Article

\title{
Some Identities Involving Two-Variable Partially Degenerate Hermite Polynomials Induced from Differential Equations and Structure of Their Roots
}

\author{
Kyung-Won Hwang ${ }^{1}$ and Cheon Seoung Ryoo ${ }^{2, *}$ \\ 1 Department of Mathematics, Dong-A University, Busan 604-714, Korea; khwang@dau.ac.kr \\ 2 Department of Mathematics, Hannam University, Daejeon 34430, Korea \\ * Correspondence: ryoocs@hnu.kr
}

Received: 23 March 2020; Accepted: 17 April 2020; Published: 20 April 2020

\begin{abstract}
In this paper, we introduce two-variable partially degenerate Hermite polynomials and get some new symmetric identities for two-variable partially degenerate Hermite polynomials. We study differential equations induced from the generating functions of two-variable partially degenerate Hermite polynomials to give identities for two-variable partially degenerate Hermite polynomials. Finally, we study the symmetric properties of the structure of the roots of the two-variable partially degenerate Hermite equations.
\end{abstract}

Keywords: differential equations; symmetric identities; partially degenerate Hermite polynomials; complex zeros

\section{Introduction}

The Hermite equation is defined as

$$
u^{\prime \prime}(x)-2 x u^{\prime}(x)+(\varepsilon-1) u(x)=0, x \in[-\infty, \infty],
$$

where $\varepsilon$ is unrestricted. The Hermite equation is encountered in the study of a quantum mechanical harmonic oscillator, where $\varepsilon$ represents the energy of the oscillator. The ordinary Hermite numbers $H_{n}$ and Hermite polynomials $H_{n}(x)$ are known by this way

$$
e^{t(2 x-t)}=\sum_{n=0}^{\infty} H_{n}(x) \frac{t^{n}}{n !}
$$

and

$$
e^{-t^{2}}=\sum_{n=0}^{\infty} H_{n} \frac{t^{n}}{n !}
$$

Clearly, $H_{n}=H_{n}(0)$. These numbers and polynomials have important roles in several areas, especially in physics, numerical analysis, combinatorics, differential equations, and so on. The Hermite polynomials are orthogonal polynomial sequences in mathematics and physics. The Hermite polynomials are the Edgeworth series in the probability area. These polynomials appear as an example of the Appell sequence. These have roles in the Gaussian quadrature in numerical analysis. These appear in the eigenstates of the quantum harmonic oscillator in physics.

It is known that these numbers and polynomials have an important role in various areas of mathematics and physics, as we mention in the above sentences. Many interesting properties about 
that have been studied (see [1-5]). The ordinary Hermite polynomials $H_{n}(x)$ have the following Hermite differential equation

$$
\frac{d^{2} H(x)}{d x^{2}}-2 x \frac{d H(x)}{d x}+2 n H(x)=0, n=0,1,2, \ldots
$$

Hence, ordinary Hermite polynomials $H_{n}(x)$ satisfy the second-order ordinary differential equation

$$
u^{\prime \prime}-2 x u^{\prime}+2 n u=0 .
$$

We remind that the two-variable Hermite polynomials $H_{n}(x, y)$ are (see [2])

$$
\sum_{n=0}^{\infty} H_{n}(x, y) \frac{t^{n}}{n !}=e^{t(x+y t)}
$$

$H_{n}(x, y)$ are the solution of the heat equation

$$
\frac{\partial}{\partial y} H_{n}(x, y)=\frac{\partial^{2}}{\partial x^{2}} H_{n}(x, y), \quad H_{n}(x, 0)=x^{n} .
$$

We can see

$$
H_{n}(2 x,-1)=H_{n}(x) .
$$

Several kinds of some special numbers and polynomials were recently studied because of their importance and potential applications in several areas (see [1-7]). The area of the degenerate Stirling, degenerate Bernoulli polynomials, degenerate Euler polynomials, degenerate Genocchi polynomials, and degenerate tangent polynomials have been studied (see [6-10]).

Recently, Hwang and Ryoo [11] proposed the two-variable degenerate Hermite polynomials $\mathcal{H}_{n}(x, y, \lambda)$ by using the generating function

$$
\sum_{n=0}^{\infty} \mathcal{H}_{n}(x, y, \lambda) \frac{t^{n}}{n !}=(1+\lambda) \frac{t(x+y t)}{\lambda} .
$$

Since $(1+\lambda)^{\frac{t}{\lambda}} \rightarrow e^{t}$ as $\lambda \rightarrow 0$, it is clear that Equation (3) can be reduced to Equation (1). The $\mathcal{H}_{n}(x, y, \lambda)$ in generating Function (3) are the solutions of the below equation

$$
\begin{aligned}
& \frac{\partial}{\partial y} \mathcal{H}_{n}(x, y, \lambda)=\frac{\lambda}{\log (1+\lambda)} \frac{\partial^{2}}{\partial x^{2}} \mathcal{H}_{n}(x, y, \lambda), \\
& \mathcal{H}_{n}(x, 0, \lambda)=\left(\frac{\log (1+\lambda)}{\lambda}\right)^{n} x^{n} .
\end{aligned}
$$

Since $\frac{\log (1+\lambda)}{\lambda} \rightarrow 1$ as $\lambda$ approaches 0 , it is apparent that Equation (4) descends to Equation (2).

The differential equations induced from the generating functions of special numbers and polynomials have been studied (see [10-16]). Now, a new class of two-variable partially degenerate Hermite polynomials is constructed based on the results so far. We can make the differential equations generated from two-variable partially degenerate Hermite polynomials. We get identities for the 2-variable partially degenerate Hermite polynomials by using the coefficients of this differential equation. The remaining parts of the paper are written as follows. In Section 2, we construct the two-variable partially degenerate Hermite polynomials and get the basic properties of these polynomials. In Section 3, we give symmetric identities for two-variable partially degenerate Hermite polynomials. In Section 4, we induce the differential equations induced from two-variable partially degenerate Hermite polynomials. We make identities for the two-variable partially degenerate Hermite 
polynomials by using the coefficients of differential equations. In Section 5, we induce the roots of the two-variable partially degenerate Hermite equations by using a computer. Furthermore, we try to find the pattern for the roots of the two-variable partially degenerate Hermite equations. Our paper will be finished in Section 6, which presents the conclusions and future directions of this work.

\section{Properties for the Two-Variable Partially Degenerate Hermite Polynomials}

In this section, a new class of the two-variable partially degenerate Hermite polynomials are considered. Furthermore, some properties of these polynomials are also made.

We define the two-variable partially degenerate Hermite polynomials $\mathbf{H}_{n}(x, y, \lambda)$ like this

$$
\sum_{n=0}^{\infty} \mathbf{H}_{n}(x, y, \lambda) \frac{t^{n}}{n !}=(1+\lambda t)^{\frac{x}{\lambda}} e^{y t^{2}}
$$

If $\lambda \rightarrow 0,(1+\lambda t)^{\frac{x}{\lambda}} \rightarrow e^{x t}$. It is clear that Equation (5) can be reduced to Equation (1). Observe that degenerate Hermite polynomials $\mathcal{H}_{n}(x, y, \lambda)$ and two-variable partially degenerate Hermite polynomials $\mathbf{H}_{n}(x, y, \lambda)$ are totally different.

Now, we recall the following formula:

$$
(x \mid \lambda)_{0}=1,(x \mid \lambda)_{n}=x(x-\lambda)(x-2 \lambda) \cdots(x-(n-1) \lambda),(n \geq 1) .
$$

As we know, $\lim _{\lambda \rightarrow 1}(x \mid \lambda)_{n}=x(x-1)(x-2) \cdots(x-(n-1))=(x)_{n},(n \geq 1)$. We recall the binomial theorem for a variable $y$.

$$
\begin{aligned}
(1+\lambda t)^{\frac{y}{\lambda}} & =\sum_{m=0}^{\infty}\left(\frac{y}{\lambda}\right)_{m} \lambda^{m} \frac{t^{m}}{m !} \\
& =\sum_{m=0}^{\infty}(y \mid \lambda)_{m} \frac{t^{m}}{m !}
\end{aligned}
$$

We remember that $S_{1}(n, k)$ and $S_{2}(n, k)$ have these relations(see [6-12])

$$
(x)_{n}=\sum_{k=0}^{n} S_{1}(n, k) x^{k} \text { and } x^{n}=\sum_{k=0}^{n} S_{2}(n, k)(x)_{k},
$$

respectively. We also have

$$
\sum_{n=m}^{\infty} S_{2}(n, m) \frac{t^{n}}{n !}=\frac{\left(e^{t}-1\right)^{m}}{m !} \text { and } \sum_{n=m}^{\infty} S_{1}(n, m) \frac{t^{n}}{n !}=\frac{(\log (1+t))^{m}}{m !} .
$$

As a different application of the differential equation for $\mathbf{H}_{n}(x, y, \lambda)$ is this: Note that

$$
G(t, x, y, \lambda)=(1+\lambda t)^{\frac{x}{\lambda}} e^{y t^{2}}
$$

satisfies

$$
\frac{\log (1+\lambda)}{\lambda} \frac{\partial G(t, x, y, \lambda)}{\partial y}-\frac{\partial^{2} G(t, x, y, \lambda)}{\partial x \partial y}=0 .
$$

If we substitute the series in Equation (5) for $G(t, x, y, \lambda)$, we get

$$
\frac{\partial^{2}}{\partial x \partial y} \mathbf{H}_{n}(x, y, \lambda)-\sum_{l=0}^{n-1} \frac{(-1)^{l} \lambda^{l} n !}{(l+1)(n-l-1) !} \frac{\partial}{\partial y} \mathbf{H}_{n-l-1}(x, y, \lambda)=0 .
$$


Thus the two-variable partially degenerate Hermite polynomials $\mathbf{H}_{n}(x, y, \lambda)$ in Equation (5) are the solution of equation

$$
\begin{aligned}
& \frac{\partial^{2}}{\partial x \partial y} \mathbf{H}_{n}(x, y, \lambda)=\sum_{l=0}^{n-1} \frac{(-1)^{l} \lambda^{l}}{(l+1)} \frac{\partial}{\partial y} \mathbf{H}_{n-l-1}(x, y, \lambda) \frac{n !}{(n-l-1) !}, \\
& \mathbf{H}_{n}(x, 0, \lambda)=(x \mid \lambda)_{n} .
\end{aligned}
$$

Then, Equation (5) is used for making several properties of the two-variable partially degenerate Hermite polynomials $\mathbf{H}_{n}(x, y, \lambda)$. For example, we have the following formula:

Theorem 1. For any positive integer $n$, we have

$$
\mathbf{H}_{n}(x, y, \lambda)=\sum_{k=0}^{\left[\frac{n}{2}\right]} y^{k}(x \mid \lambda)_{n-2 k} \frac{n !}{k !(n-2 k) !},
$$

where [ ] denotes taking the integer part.

Proof 1. By Equations (5) and (6), we have

$$
\begin{aligned}
\sum_{n=0}^{\infty} \mathbf{H}_{n}(x, y, \lambda) \frac{t^{n}}{n !} & =(1+\lambda t) \frac{x}{\lambda} e^{y t^{2}} \\
& =\sum_{k=0}^{\infty} y^{k} \frac{t^{2 k}}{k !} \sum_{l=0}^{\infty}(x \mid \lambda)_{l} \frac{t^{l}}{l !} \\
& =\sum_{n=0}^{\infty}\left(\sum_{k=0}^{\left[\frac{n}{2}\right]} y^{k}(x \mid \lambda)_{n-2 k} \frac{n !}{k !(n-2 k) !}\right) \frac{t^{n}}{n !} .
\end{aligned}
$$

By comparing the coefficients of $\frac{t^{n}}{n !}$, we get Theorem 1 like this.

Since $\lim _{\lambda \rightarrow 0}(x \mid \lambda)_{n}=x^{n},(n \geq 1)$, we get

$$
H_{n}(x, y)=n ! \sum_{k=0}^{\left[\frac{n}{2}\right]} \frac{y^{k} x^{n-2 k}}{k !(n-2 k) !} .
$$

The following properties of $\mathbf{H}_{n}(x, y, \lambda)$ are induced from Equation (5). Therefore, it is enough to delete the involved detail explanation.

Theorem 2. For any positive integer $n$, we have

$$
\begin{aligned}
& \text { (1) } \mathbf{H}_{n}(x, y, \lambda)=\sum_{k=0}^{\left[\frac{n}{2}\right]} \sum_{l=0}^{n-2 k} y^{k} S_{1}(n-2 k, l) x^{l} \lambda^{n-2 k-l} \frac{n !}{k !(n-2 k) !} \\
& \text { (2) } \mathbf{H}_{n}\left(x_{1}+x_{2}, y, \lambda\right)=\sum_{l=0}^{n}\left(\begin{array}{c}
n \\
l
\end{array}\right)\left(x_{2} \mid \lambda\right)_{l} \mathbf{H}_{n-l}\left(x_{1}, y, \lambda\right) . \\
& \text { (3) } \mathbf{H}_{n}\left(x_{1}+x_{2}, y, \lambda\right)=\sum_{k=0}^{n} \sum_{l=0}^{n-k}\left(\begin{array}{l}
n \\
k
\end{array}\right) \mathbf{H}_{k}\left(x_{1}, y, \lambda\right) S_{1}(n-k, l) x_{2}^{l} \lambda^{n-k-l} . \\
& \text { (4) } \mathbf{H}_{n}\left(x, y_{1}+y_{2}, \lambda\right)=\sum_{k=0}^{\left[\frac{n}{2}\right]} \mathbf{H}_{k}\left(x, y_{1}, \lambda\right) y_{2}^{n-2 k} \frac{n !}{k !(n-2 k) !} . \\
& \text { (5) } \mathbf{H}_{n}\left(x_{1}+x_{2}, y_{1}+y_{2}, \lambda\right)=\sum_{l=0}^{n}\left(\begin{array}{c}
n \\
l
\end{array}\right) \mathbf{H}_{l}\left(x_{1}, y_{1}, \lambda\right) \mathbf{H}_{n-l}\left(x_{2}, y_{2}, \lambda\right) .
\end{aligned}
$$




\section{Symmetric Identities for the Two-Variable Partially Degenerate Hermite Polynomials}

In this section, new symmetric identities about the two-variable partially degenerate Hermite polynomials are given. Some formulas and properties about the two-variable partially degenerate Hermite polynomials are made.

Theorem 3. Let $a, b>0(a \neq b)$. The following identity holds true:

$$
a^{m} \mathbf{H}_{m}\left(b x, b^{2} y, \frac{\lambda}{a}\right)=b^{m} \mathbf{H}_{m}\left(a x, a^{2} y, \frac{\lambda}{b}\right) .
$$

Proof 2. Let $a, b>0(a \neq b)$. We start with

$$
\mathcal{G}(t, \lambda)=(1+\lambda t) \frac{a b x}{\lambda} e^{a^{2} b^{2} y t^{2}} .
$$

Then, the formula for $\mathcal{G}(t, \lambda)$ is symmetric in $a$ and $b$ as we see

$$
\mathcal{G}(t, \lambda)=\sum_{m=0}^{\infty} \mathbf{H}_{m}\left(a x, a^{2} y, \frac{\lambda}{b}\right) \frac{(b t)^{m}}{m !}=\sum_{m=0}^{\infty} b^{m} \mathbf{H}_{m}\left(a x, a^{2} y, \frac{\lambda}{b}\right) \frac{t^{m}}{m !} .
$$

By the same way, we get the below formula

$$
\mathcal{G}(t, \lambda)=\sum_{m=0}^{\infty} \mathbf{H}_{m}\left(b x, b^{2} y, \frac{\lambda}{a}\right) \frac{(a t)^{m}}{m !}=\sum_{m=0}^{\infty} a^{m} \mathbf{H}_{m}\left(b x, b^{2} y, \frac{\lambda}{a}\right) \frac{t^{m}}{m !} .
$$

If we compare the coefficients of $\frac{t^{m}}{m !}$ in last two equations, then the expected result of Theorem 1 is achieved.

Again, we now use

$$
\mathcal{F}(t, \lambda)=\frac{a b t(1+\lambda t) \frac{a b x}{\lambda} e^{a^{2} b^{2} y t^{2}}\left((1+\lambda t)^{\frac{a b}{\lambda}}-1\right)}{\left((1+\lambda t)^{\frac{a}{\lambda}}-1\right)\left((1+\lambda t)^{\frac{b}{\lambda}}-1\right)} .
$$

For $\lambda \in \mathbb{C}$, Carlitz introduced the degenerate Bernoulli polynomials like the below formula $($ see $[6,7])$

$$
\sum_{n=0}^{\infty} \beta_{n}(x, \lambda) \frac{t^{n}}{n !}=\frac{t}{(1+\lambda t)^{\frac{1}{\lambda}}-1}(1+\lambda t)^{\frac{x}{\lambda}} .
$$

When $x=0$ and $\beta_{n}(\lambda)=\beta_{n}(0, \lambda)$ are the degenerate Bernoulli numbers as we know. We refer that

$$
\lim _{\lambda \rightarrow 0} \beta_{n}(\lambda)=B_{n}
$$

where $B_{n}$ are the Bernoulli numbers (see $\left.[6,7,17]\right)$. 
For each integer $k \geq 0, \mathbf{S}_{k}(n)=0^{k}+1^{k}+2^{k}+\cdots+(n-1)^{k}$ is sum of integers. A generalized falling factorial sum $\sigma_{k}(n, \lambda)$ is this (see $[6,7,9,17]$ )

$$
\sum_{k=0}^{\infty} \sigma_{k}(n, \lambda) \frac{t^{k}}{k !}=\frac{(1+\lambda t)^{\frac{(n+1)}{\lambda}}-1}{(1+\lambda t)^{\frac{1}{\lambda}}-1} .
$$

Note that $\lim _{\lambda \rightarrow 0} \sigma_{k}(n, \lambda)=\mathbf{S}_{k}(n)$. From $\mathcal{F}(t, \lambda)$, we get the below formula:

$$
\begin{gathered}
\mathcal{F}(t, \lambda)=\frac{a b t(1+\lambda t)^{\frac{a b x}{\lambda}} e^{a^{2} b^{2} y t^{2}}\left((1+\lambda t)^{\frac{a b}{\lambda}}-1\right)}{\left((1+\lambda t)^{\frac{a}{\lambda}}-1\right)\left((1+\lambda t) \frac{b}{\lambda}-1\right)} \\
=\frac{a b t}{\left((1+\lambda t)^{\frac{a}{\lambda}}-1\right)}(1+\lambda t) \frac{a b x}{\lambda} e^{a^{2} b^{2} y t^{2}} \frac{\left((1+\lambda t) \frac{a b}{\lambda}-1\right)}{\left((1+\lambda t) \frac{b}{\lambda}-1\right)} \\
=b \sum_{n=0}^{\infty} \beta_{n}\left(\frac{\lambda}{a}\right) \frac{(a t)^{n}}{n !} \sum_{n=0}^{\infty} \mathbf{H}_{n}\left(b x, b^{2} y, \frac{\lambda}{a}\right) \frac{(a t)^{n}}{n !} \sum_{n=0}^{\infty} \sigma_{k}\left(a-1, \frac{\lambda}{b}\right) \frac{(b t)^{n}}{n !} \\
=\sum_{n=0}^{\infty}\left(\sum_{i=0}^{n} \sum_{m=0}^{i}\left(\begin{array}{c}
n \\
i
\end{array}\right)\left(\begin{array}{c}
i \\
m
\end{array}\right) a^{i} b^{n+1-i} \beta_{m}\left(\frac{\lambda}{a}\right) \mathbf{H}_{i-m}\left(b x, b^{2} y, \frac{\lambda}{a}\right) \sigma_{n-i}\left(a-1, \frac{\lambda}{b}\right)\right) \frac{t^{n}}{n !} .
\end{gathered}
$$

In a similar fashion, we have

$$
\begin{aligned}
\mathcal{F}(t, \lambda) & =\frac{a b t}{\left((1+\lambda t)^{\frac{b}{\lambda}}-1\right)}(1+\lambda t) \frac{a b x}{\lambda} e^{a^{2} b^{2} y t^{2}} \frac{\left((1+\lambda t)^{\frac{a b}{\lambda}}-1\right)}{\left((1+\lambda t)^{\frac{a}{\lambda}}-1\right)} \\
& =a \sum_{n=0}^{\infty} \beta_{n}\left(\frac{\lambda}{b}\right) \frac{(b t)^{n}}{n !} \sum_{n=0}^{\infty} \mathbf{H}_{n}\left(a x, a^{2} y, \frac{\lambda}{b}\right) \frac{(b t)^{n}}{n !} \sum_{n=0}^{\infty} \sigma_{k}\left(b-1, \frac{\lambda}{a}\right) \frac{(a t)^{n}}{n !} \\
& =\sum_{n=0}^{\infty}\left(\sum_{i=0}^{n} \sum_{m=0}^{i}\left(\begin{array}{c}
n \\
i
\end{array}\right)\left(\begin{array}{c}
i \\
m
\end{array}\right) b^{i} a^{n+1-i} \beta_{m}\left(\frac{\lambda}{b}\right) \mathbf{H}_{i-m}\left(a x, a^{2} y, \frac{\lambda}{b}\right) \sigma_{n-i}\left(b-1, \frac{\lambda}{a}\right)\right) \frac{t^{n}}{n !}
\end{aligned}
$$

If we compare the coefficients of $\frac{t^{m}}{m !}$ on the right hand sides of the last two equations, we have the below theorem.

Theorem 4. Let $a, b>0$ for $a \neq b$. We have the below identity:

$$
\begin{aligned}
& \sum_{i=0}^{n} \sum_{m=0}^{i}\left(\begin{array}{c}
n \\
i
\end{array}\right)\left(\begin{array}{c}
i \\
m
\end{array}\right) a^{i} b^{n+1-i} \beta_{m}\left(\frac{\lambda}{a}\right) \mathbf{H}_{i-m}\left(b x, b^{2} y, \frac{\lambda}{a}\right) \sigma_{n-i}\left(a-1, \frac{\lambda}{b}\right) \\
& =\sum_{i=0}^{n} \sum_{m=0}^{i}\left(\begin{array}{c}
n \\
i
\end{array}\right)\left(\begin{array}{c}
i \\
m
\end{array}\right) b^{i} a^{n+1-i} \beta_{m}\left(\frac{\lambda}{b}\right) \mathbf{H}_{i-m}\left(a x, a^{2} y, \frac{\lambda}{b}\right) \sigma_{n-i}\left(b-1, \frac{\lambda}{a}\right) .
\end{aligned}
$$


By taking the limit as $\lambda \rightarrow 0$, we have the following corollary.

Corollary 1. Let $a, b>0(a \neq b)$. We have this:

$$
\begin{aligned}
& \sum_{i=0}^{n} \sum_{m=0}^{i}\left(\begin{array}{c}
n \\
i
\end{array}\right)\left(\begin{array}{c}
i \\
m
\end{array}\right) a^{i} b^{n+1-i} B_{m} H_{i-m}\left(b x, b^{2} y\right) \mathbf{S}_{n-i}(a-1) \\
& =\sum_{i=0}^{n} \sum_{m=0}^{i}\left(\begin{array}{c}
n \\
i
\end{array}\right)\left(\begin{array}{c}
i \\
m
\end{array}\right) b^{i} a^{n+1-i} B_{m} H_{i-m}\left(a x, a^{2} y\right) \mathbf{S}_{n-i}(b-1) .
\end{aligned}
$$

\section{Differential Equations Related to Two-Variable Partially Degenerate Hermite Polynomials}

In this section, we construct the differential equations with coefficients $a_{i}(N, x, y, \lambda)$ induced from the two-variable partially degenerate Hermite polynomials:

$$
\begin{aligned}
& \left(\frac{\partial}{\partial t}\right)^{N} G(t, x, y, \lambda)-a_{0}(N, x, y, \lambda)(1+\lambda t)^{-N} G(t, x, y, \lambda)-\cdots \\
& -a_{2 N}(N, x, y, \lambda)(1+\lambda t)^{-N} t^{2 N} G(t, x, y, \lambda)=0 .
\end{aligned}
$$

We get identities for the two-variable partially degenerate Hermite polynomials $\mathbf{H}_{n}(x, y, \lambda)$ when we compare the coefficients of differential equations. Remember that

$$
\begin{aligned}
G & =G(t, x, y, \lambda) \\
& =(1+\lambda t) \frac{x}{\lambda} e^{y t^{2}} \\
& =\sum_{n=0}^{\infty} \mathbf{H}_{n}(x, y, \lambda) \frac{t^{n}}{n !}, \quad \lambda, x, t \in \mathbb{C} .
\end{aligned}
$$

From Equation (7), we get

$$
\begin{gathered}
G^{(1)}=\frac{\partial}{\partial t} G(t, x, y, \lambda) \\
=\frac{\partial}{\partial t}\left((1+\lambda t) \frac{x}{\lambda} e^{y t^{2}}\right) \\
=\left(\frac{x}{1+\lambda t}+2 y t\right)(1+\lambda t) \frac{x}{\lambda} e^{y t^{2}} \\
=\left(\frac{x+2 y t+2 y \lambda t^{2}}{1+\lambda t}\right) G(t, x, y, \lambda), \\
G^{(2)}=\frac{\partial}{\partial t} G^{(1)}(t, x, y, \lambda) \\
=\left(\frac{(2 y+4 y \lambda t)(1+\lambda t)-\left(x+2 y t+2 y \lambda t^{2}\right) \lambda}{(1+\lambda t)^{2}}\right) G(t, x, y, \lambda) \\
+\left(\frac{x+2 y t+2 y \lambda t^{2}}{1+\lambda t}\right) G^{(1)}(t, x, y, \lambda)
\end{gathered}
$$




$$
\begin{aligned}
& =\left(\frac{2 y-\lambda x+x^{2}}{(1+\lambda t)^{2}}\right) G(t, x, y, \lambda) \\
& \quad+\left(\frac{4 x y}{(1+\lambda t)^{2}}\right) t G(t, x, y, \lambda) \\
& \quad+\left(\frac{4 \lambda^{2} y+4 \lambda x y+4 y^{2}-2 \lambda^{2} y}{(1+\lambda t)^{2}}\right) t^{2} G(t, x, y, \lambda) \\
& \quad+\left(\frac{8 \lambda y^{2}}{(1+\lambda t)^{2}}\right) t^{3} G(t, x, y, \lambda) \\
& \quad+\left(\frac{4 \lambda^{2} y^{2}}{(1+\lambda t)^{2}}\right) t^{4} G(t, x, y, \lambda) .
\end{aligned}
$$

When we do this process continuously, as shown in (9), we easily get this

$$
\begin{aligned}
G^{(N)} & =\left(\frac{\partial}{\partial t}\right)^{N} G(t, x, y, \lambda) \\
& =\sum_{i=0}^{2 N} a_{i}(N, x, y, \lambda)(1+\lambda t)^{-N} t^{i} G(t, x, y, \lambda),(N=0,1,2, \ldots) .
\end{aligned}
$$

If we differentiate Equation (10) with respect to $t$, we get

$$
\begin{aligned}
G^{(N+1)}= & \frac{\partial G(N)}{\partial t}=\sum_{i=0}^{2 N} a_{i}(N, x, y, \lambda)(i) t^{i-1}(1+\lambda t)^{-N} G(t, x, y, \lambda) \\
& \quad+\sum_{i=0}^{2 N} a_{i}(N, x, y, \lambda) t^{i}(-N) \lambda(1+\lambda t)^{-N-1} G(t, x, y, \lambda) \\
& +\sum_{i=0}^{2 N} a_{i}(N, x, y, \lambda) t^{i}(1+\lambda t)^{-N} G^{(1)}(t, x, y, \lambda) \\
= & \sum_{i=0}^{2 N}(i) a_{i}(N, x, y, \lambda) t^{i-1}(1+\lambda t)^{-(N+1)} G(t, x, y, \lambda) \\
& +\sum_{i=0}^{2 N}(x-\lambda N+i \lambda) a_{i}(N, x, y, \lambda) t^{i}(1+\lambda t)^{-(N+1)} G(t, x, y, \lambda) \\
& +\sum_{i=0}^{2 N}(2 y) a_{i}(N, x, y, \lambda) t^{i+1}(1+\lambda t)^{-(N+1)} G(t, x, y, \lambda) \\
& +\sum_{i=0}^{2 N}(2 \lambda y) a_{i}(N, x, y, \lambda) t^{i+2}(1+\lambda t)^{-(N+1)} G(t, x, y, \lambda) \\
& +\sum_{i=1}^{2 N+2}(2 \lambda y) a_{i-2}(N, x, y, \lambda) t^{i}(1+\lambda t)^{-(N+1)} g(t, x, y, \lambda) \\
= & \sum_{i=0}^{2 N}(i+1) a_{i+1}(N, x, y, \lambda) t^{i}(1+\lambda t)^{-(N+1)} G(t, x, y, \lambda) \\
& +\sum_{i=0}^{2 N}(x-\lambda N+i \lambda) a_{i}(N, x, y, \lambda) t^{i}(1+\lambda t)^{-(N+1)} G(t, x, y, \lambda) \\
&
\end{aligned}
$$


Now we replace $N+1$ instead of $N$ in Equation (10). We find

$$
G^{(N+1)}=\sum_{i=0}^{2 N+2} a_{i}(N+1, x, y, \lambda) t^{i}(1+\lambda t)^{-(N+1)} G(t, x, y, \lambda) .
$$

If we compare the coefficients on both sides of Equations (11) and (12), we get

$$
\begin{aligned}
a_{0}(N+1, x, y, \lambda)= & a_{1}(N, x, y, \lambda)+(x-\lambda N) a_{0}(N, x, y, \lambda) . \\
a_{1}(N+1, x, y, \lambda)= & 2 a_{2}(N, x, y, \lambda)+(x-\lambda N+\lambda) a_{1}(N, x, y, \lambda) \\
& +(2 y) a_{0}(N, x, y, \lambda) .
\end{aligned}
$$

For $2 \leq i \leq 2 N-1$, we make

$$
\begin{aligned}
a_{i}(N+1, x, y, \lambda)= & (i+1) a_{i+1}(N, x, y, \lambda)+(x-\lambda N+i \lambda) a_{i}(N, x, y, \lambda) \\
& +(2 y) a_{i-1}(N, x, y, \lambda)+(2 \lambda y) a_{i-2}(N, x, y, \lambda) .
\end{aligned}
$$

For $i=2 N$, we obtain

$$
\begin{aligned}
a_{2 N}(N+1, x, y, \lambda) & =(x-\lambda N+2 \lambda N) a_{2 N}(N, x, y, \lambda) \\
& +(2 y) a_{2 N-1}(N, x, y, \lambda)+(2 \lambda y) a_{2 N-2}(N, x, y, \lambda) .
\end{aligned}
$$

For $i=2 N+1$, we obtain

$$
a_{2 N+1}(N+1, x, y, \lambda)=(2 y) a_{2 N}(N, x, y, \lambda)+(2 \lambda y) a_{2 N-1}(N, x, y, \lambda) .
$$

For $i=2 N+2$, we obtain

$$
a_{2 N+2}(N+1, x, y, \lambda)=(2 \lambda y) a_{2 N}(N, x, y, \lambda) .
$$

We also have the below identity from Equation (10)

$$
G(t, x, y, \lambda)=G^{(0)}(t, x, y, \lambda)=a_{0}(0, x, y, \lambda) G(t, x, y, \lambda) .
$$

By Equation (19), we easily have

$$
a_{0}(0, x, y, \lambda)=1
$$

It is easy to see this

$$
\begin{aligned}
x & (1+\lambda t)^{-1} G(t, x, y, \lambda)+2 y t(1+\lambda t)^{-1} G(t, x, y, \lambda)+2 y \lambda t^{2}(1+\lambda t)^{-1} G(t, x, y, \lambda) \\
= & G^{(1)}(t, x, y, \lambda) \\
= & \sum_{i=0}^{2} a_{i}(1, x, y, \lambda) t^{i}(1+\lambda t)^{-1} G(t, x, y, \lambda) \\
= & a_{0}(1, x, y, \lambda)(1+\lambda t)^{-1} G(t, x, y, \lambda)+a_{1}(1, x, y, \lambda) t(1+\lambda t)^{-1} G(t, x, y, \lambda) \\
& +a_{2}(1, x, y, \lambda) t^{2}(1+\lambda t)^{-1} G(t, x, y, \lambda) .
\end{aligned}
$$

From Equations (8) and (21), we also get

$$
a_{0}(1, x, y, \lambda)=x, \quad a_{1}(1, x, y, \lambda)=2 y, \quad a_{2}(1, x, y, \lambda)=2 \lambda y .
$$


From Equation (13), we see this

$$
\begin{aligned}
& a_{0}(N+1, x, y, \lambda)=a_{1}(N, x, y, \lambda)+(x-\lambda N) a_{0}(N, x, y, \lambda), \\
& a_{0}(N, x, y, \lambda)=a_{1}(N-1, x, y, \lambda)+(x-\lambda(N-1)) a_{0}(N-1, x, y, \lambda), \\
& \ldots \\
& a_{0}(N+1, x, y, \lambda)=\sum_{i=0}^{N}(x \mid \lambda)_{i}^{(N)} a_{1}(N-i, x, y, \lambda)+(x \mid \lambda)_{N+1}^{(N)},
\end{aligned}
$$

where $(x \mid \lambda)_{0}^{(N)}=1,(x \mid \lambda)_{i}^{(N)}=(x-\lambda N) \cdots(x-\lambda(N+1-i))$.

From Equation (16), we get

$$
\begin{aligned}
a_{2 N}(N+1, x, y, \lambda) & =(x+N \lambda) a_{2 N}(N, x, y, \lambda)+(2 y) a_{2 N-1}(N, x, y, \lambda) \\
& +(2 \lambda y) a_{2 N-2}(N, x, y, \lambda), \\
a_{2 N-2}(N, x, y, \lambda)= & (x+(N-1) \lambda) a_{2 N-2}(N-1, x, y, \lambda) \\
& +(2 y) a_{2 N-3}(N-1, x, y, \lambda) \\
& +(2 \lambda y) a_{2 N-4}(N-1, x, y, \lambda), \ldots \\
a_{2 N}(N+1, x, y, \lambda)= & \sum_{i=0}^{N}(2 \lambda y)^{i}(x+\lambda(N-i)) a_{2 N-2 i}(N-i, x, y, \lambda) \\
& +(2 y) \sum_{i=0}^{N-1}(2 \lambda y)^{i} a_{2 N-(2 i+1)}(N-i, x, y, \lambda) .
\end{aligned}
$$

By Equation (17), we get

$$
\begin{aligned}
& a_{2 N+1}(N+1, x, y, \lambda)=(2 y) a_{2 N}(N, x, y, \lambda)+(2 \lambda y) a_{2 N-1}(N, x, y, \lambda), \\
& a_{2 N-1}(N, x, y, \lambda)=(2 y) a_{2 N-2}(N-1, x, y, \lambda)+(2 \lambda y) a_{2 N-3}(N-1, x, y, \lambda), \\
& \ldots \\
& a_{2 N+1}(N+1, x, y, \lambda)=(2 y)(2 y \lambda)^{N} .
\end{aligned}
$$

Again, by Equation (14), we make

$$
\begin{aligned}
a_{1}(N+1, x, y, \lambda)= & 2 \sum_{k=0}^{N}(x \mid \lambda)_{k}^{(N-1)} a_{2}(N-k, x, y, \lambda) \\
& +2 y \sum_{k=0}^{N}(x \mid \lambda)_{k}^{(N-1)} a_{0}(N-k, x, y, \lambda),
\end{aligned}
$$

From Equation (18), we have

$$
\begin{aligned}
& a_{2 N+2}(N+1, x, y, \lambda)=(2 y \lambda) a_{2 N}(N, x, y, \lambda), \\
& a_{2 N}(N, x, y, \lambda)=(2 y \lambda) a_{2 N-2}(N-1, x, y, \lambda), \ldots \\
& a_{2 N+2}(N+1, x, y, \lambda)=(2 y \lambda)^{N+1} .
\end{aligned}
$$

We do this process continuously. We get the below formula for $2 \leq i \leq 2 N-1$, 


$$
\begin{aligned}
a_{i}(N+1, x, y, \lambda)= & (i+1) \sum_{k=0}^{N}(x \mid \lambda)_{i}^{(N-i)} a_{i+1}(N-k, x, y, \lambda) \\
& +2 y \sum_{k=0}^{N}(x \mid \lambda)_{k}^{(N-i)} a_{i-1}(N-k, x, y, \lambda) \\
& +2 \lambda y \sum_{k=0}^{N}(x \mid \lambda)_{k}^{(N-i)} a_{i-2}(N-k, x, y, \lambda)
\end{aligned}
$$

We get this, where the matrix $a_{i}(N, x, y, \lambda)_{0 \leq i \leq 2 N+2,0 \leq j \leq N+1}$ is given by

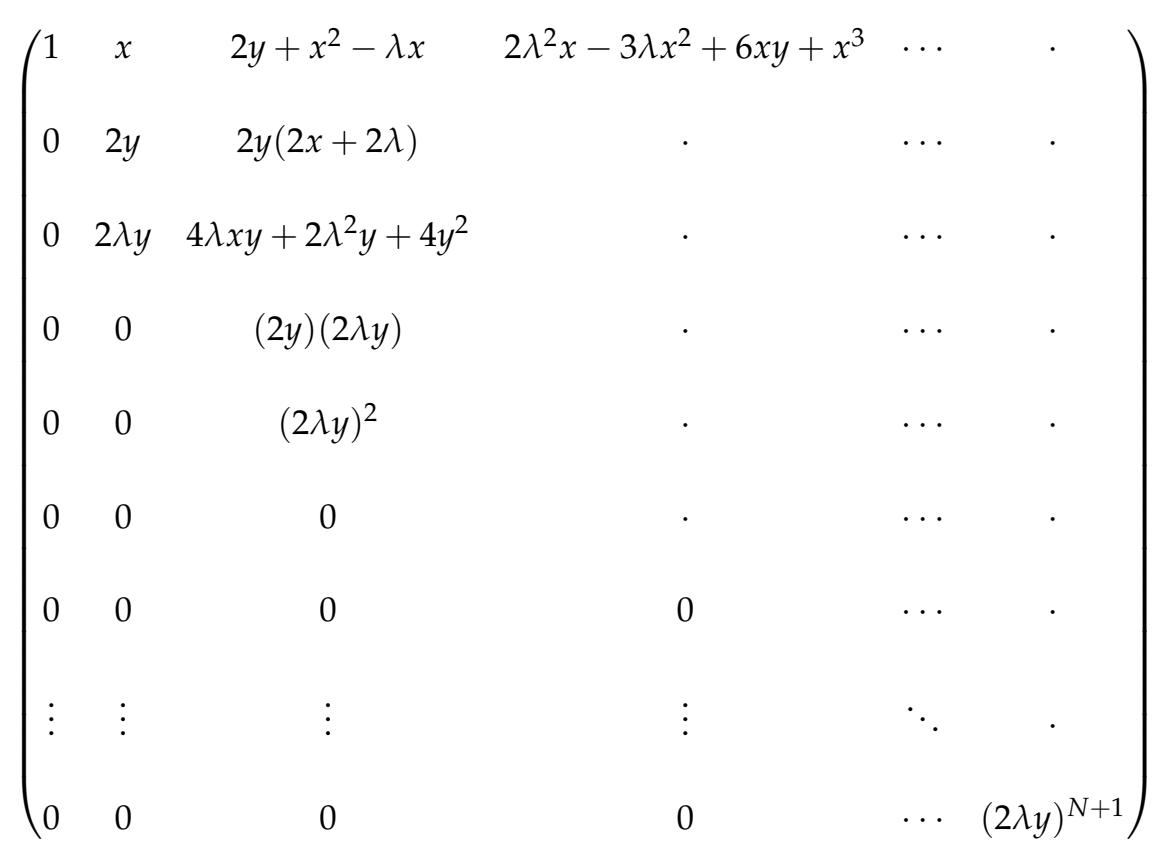

Therefore, by Equations (20)-(28), we get the below theorem.

Theorem 5. For $N=0,1,2, \ldots$, the differential equation

$$
\left(\frac{\partial}{\partial t}\right)^{N} G(t, x, y, \lambda)-\sum_{i=0}^{N} a_{i}(N, x, y, \lambda)(1+\lambda t)^{-N} t^{i} G(t, x, y, \lambda)=0
$$

has a solution

$$
G=G(t, x, y, \lambda)=(1+\lambda t)^{\frac{x}{\lambda}} e^{y t^{2}}
$$


where

$$
\begin{aligned}
& a_{0}(N+1, x, y, \lambda)= \sum_{i=0}^{N}(x \mid \lambda)_{i}^{(N)} a_{1}(N-i, x, y, \lambda)+(x \mid \lambda)_{N+1^{\prime}}^{(N)} \\
& a_{1}(N+1, x, y, \lambda)= 2 \sum_{k=0}^{N}(x \mid \lambda)_{k}^{(N-1)} a_{2}(N-k, x, y, \lambda)+2 y \sum_{k=0}^{N}(x \mid \lambda)_{k}^{(N-1)} a_{0}(N-k, x, y, \lambda) \\
& a_{2 N}(N+1, x, y, \lambda)= \sum_{i=0}^{N}(2 \lambda y)^{i}(x+\lambda(N-i)) a_{2 N-2 i}(N-i, x, y, \lambda) \\
&+(2 y) \sum_{i=0}^{N-1}(2 \lambda y)^{i} a_{2 N-(2 i+1)}(N-i, x, y, \lambda) \\
& a_{2 N+1}(N+1, x, y, \lambda)=(2 y)(2 y \lambda)^{N} \\
& a_{2 N+2}(N+1, x, y, \lambda)=(2 y \lambda)^{N+1}, \cdots \\
& a_{i}(N+1, x, y, \lambda)=(i+1) \sum_{k=0}^{N}(x \mid \lambda)_{i}^{(N-i)} a_{i+1}(N-k, x, y, \lambda) \\
&+2 y \sum_{k=0}^{N}(x \mid \lambda)_{k}^{(N-i)} a_{i-1}(N-k, x, y, \lambda) \\
&+2 \lambda y \sum_{k=0}^{N}(x \mid \lambda)_{k}^{(N-i)} a_{i-2}(N-k, x, y, \lambda),(2 \leq i \leq 2 N-1) .
\end{aligned}
$$

We have a picture of the surface for this solution. In the left picture of Figure 1 , we choose $-2 \leq x \leq$ $2,-\frac{1}{2} \leq t \leq \frac{1}{2}, \lambda=1 / 2$, and $y=2$.

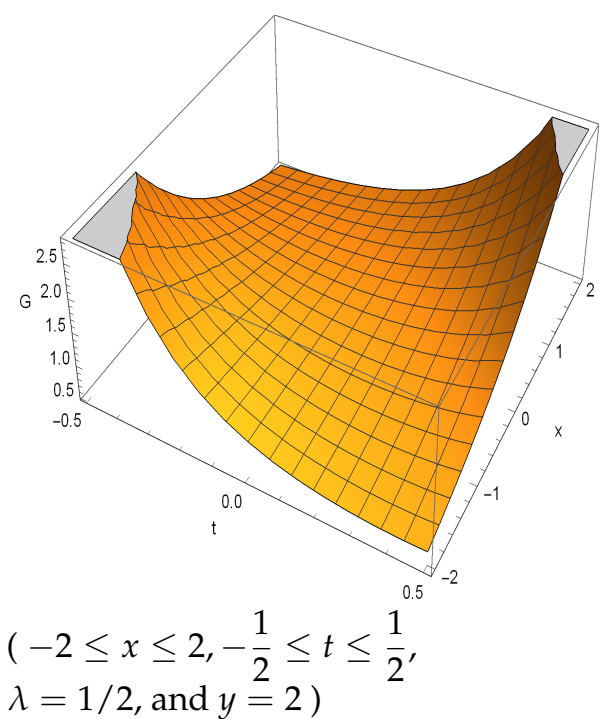

Figure 1. The surface for the solution $G(t, x, y, \lambda)=0$.

In the right picture of Figure 1 , we select $-2 \leq y \leq 2,-\frac{1}{2} \leq t \leq \frac{1}{2}, \lambda=1 / 2$, and $x=2$.

When we take $N$-times derivative for Equation (5) with respect to $t$, we get

$$
\left(\frac{\partial}{\partial t}\right)^{N} G(t, x, y, \lambda)=\sum_{m=0}^{\infty} \mathbf{H}_{m+N}(x, y, \lambda) \frac{t^{m}}{m !} .
$$


By Equation (29) and Theorem 6, we make

$$
\begin{aligned}
& a_{0}(N, x, y, \lambda)(1+\lambda t)^{-N} G(t, x, y, \lambda) \\
& +a_{1}(N, x, y, \lambda)(1+\lambda t)^{-N} t G(t, x, y, \lambda) \\
& +\cdots \\
& +a_{2 N-1}(N, x, y, \lambda)(1+\lambda t)^{-N} t^{2 N-1} G(t, x, y, \lambda) \\
& +a_{2 N}(N, x, y, \lambda)(1+\lambda t)^{-N} t^{2 N} G(t, x, y, \lambda) \\
& =\sum_{m=0}^{\infty} \mathbf{H}_{m+N}(x, y, \lambda) \frac{t^{m}}{m !} .
\end{aligned}
$$

So we make the below formula.

Theorem 6. For $N=0,1,2, \ldots$, we get

$$
\sum_{k=0}^{m}\left(\begin{array}{l}
m \\
k
\end{array}\right)(N)_{k} \lambda^{k} \mathbf{H}_{N+m-k}(x, y, \lambda)=\sum_{i=0}^{m} \frac{\mathbf{H}_{m-i}(x, y, \lambda) a_{i}(N, x, y, \lambda) m !}{(m-i) !}
$$

When we make $m=0$ from Equation (30), then we make the below corollary.

Corollary 2. We have below formula for $N=0,1,2, \ldots$

$$
\mathbf{H}_{N}(x, y, \lambda)=a_{0}(N, x, y, \lambda) \mathbf{H}_{0}(x, y, \lambda)=a_{0}(N, x, y, \lambda)
$$

where

$$
\begin{aligned}
& a_{0}(0, x, y, \lambda)=1 \\
& a_{0}(N, x, y, \lambda)=\sum_{i=0}^{N-1}(x \mid \lambda)_{i}^{(N-1)} a_{1}(N-1-i, x, y, \lambda)+(x \mid \lambda)_{N}^{(N-1)}
\end{aligned}
$$

The first few formula of them are

$$
\begin{aligned}
& \mathbf{H}_{0}(x, y, \lambda)=1 \\
& \mathbf{H}_{1}(x, y, \lambda)=x \\
& \mathbf{H}_{2}(x, y, \lambda)=-\lambda x+x^{2}+2 y \\
& \mathbf{H}_{3}(x, y, \lambda)=2 \lambda^{2} x-3 \lambda x^{2}+x^{3}+6 x y \\
& \mathbf{H}_{4}(x, y, \lambda)=-6 \lambda^{3} x+11 \lambda^{2} x^{2}-6 \lambda x^{3}+x^{4}-12 \lambda x y+12 x^{2} y+12 y^{2}, \\
& \mathbf{H}_{5}(x, y, \lambda)=24 \lambda^{4} x-50 \lambda^{3} x^{2}+35 \lambda^{2} x^{3}-10 \lambda x^{4}+x^{5}+40 \lambda^{2} x y-60 \lambda x^{2} y \\
&+20 x^{3} y+60 x y^{2} .
\end{aligned}
$$

\section{Roots of the Two-Variable Partially Degenerate Hermite Polynomials}

In this section, we would like to show some pattern for the roots of the two-variable partially degenerate Hermite equations $\mathbf{H}_{n}(x, y, \lambda)=0$ for given $n, y, \lambda$ using numerical experiments. The two-variable partially degenerate Hermite polynomials $\mathbf{H}_{n}(x, y, \lambda)$ can be realized explicitly by using a computer. We will look at the roots of the two-variable partially degenerate Hermite equations $\mathbf{H}_{n}(x, y, \lambda)=0$ for given $n, y, \lambda$. The roots of the $\mathbf{H}_{n}(x, y, \lambda)=0$ for $n=40, y=$ $2,-2,2+i,-2-i, \lambda=1 / 2$, and $x \in \mathbb{C}$ are displayed in Figure 2 .

For the top left picture in Figure 2, we select $n=40$ and $y=2$. For the top right picture in Figure 2, we select $n=40$ and $y=-2$. For the bottom left picture in Figure 2, we select $n=40$ and $y=-2+i$. For the bottom right picture in Figure 2, we select $n=40$ and $y=-2-i$. We show a distribution of roots of equations $\mathbf{H}_{n}(x, y, \lambda)=0$ for $1 \leq n \leq 50, \lambda=1 / 2$ by using a 3 -D structure in Figure 3 . 

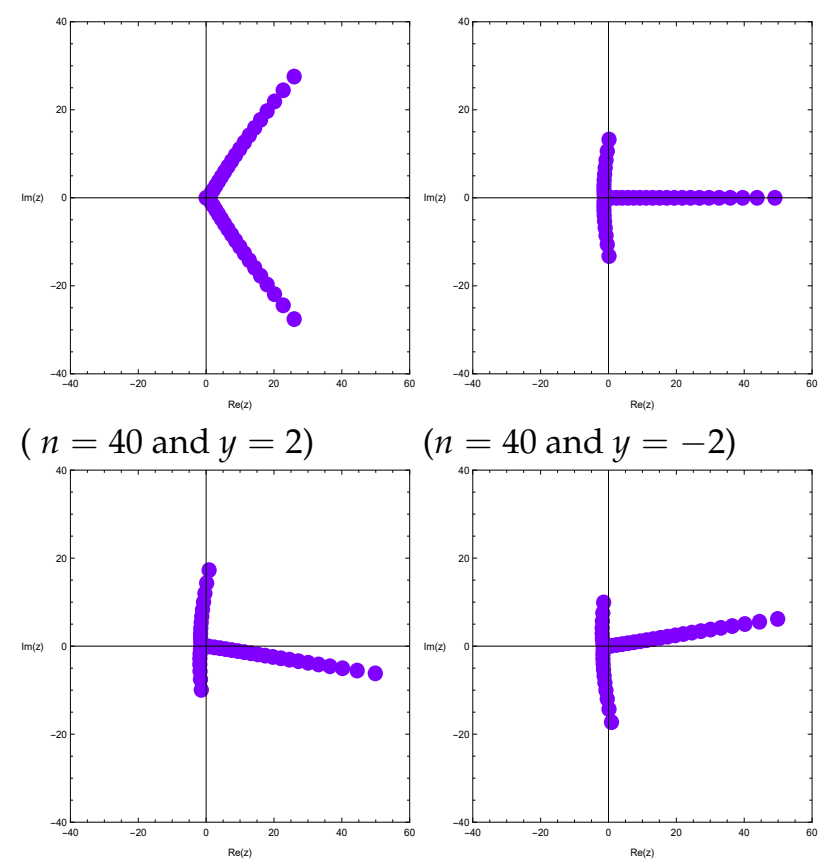

$(n=40$ and $y=-2+i) \quad(n=40$ and $y=-2-i)$

Figure 2. Roots of $\mathbf{H}_{n}(x, y, \lambda)=0$.
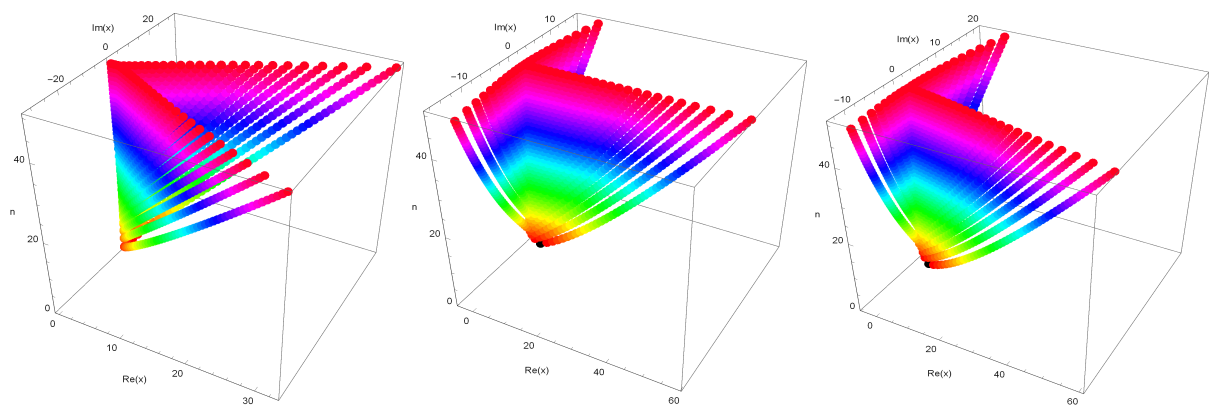

$(y=2)$

$(y=-2)$

$(y=-2+i)$

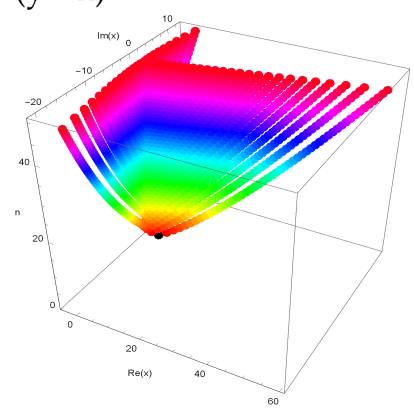

$(y=-2-i)$

Figure 3. Stacks of roots of $\mathbf{H}_{n}(x, y, \lambda)=0,1 \leq n \leq 50$.

For the top left picture in Figure 3, we select $y=2$. For the top right picture in Figure 3, we select $y=-2$. For the bottom left picture in Figure 3, we select $y=-2+i$. For the bottom right picture in Figure 3, we select $y=-2-i$.

We show our numerical experiments for approximate solutions of real roots of the two-variable partially degenerate Hermite equations $\mathbf{H}_{n}(x, y, \lambda)=0$ (Tables 1 and 2). 
Table 1. Numbers of real and complex roots of $\mathbf{H}_{n}(x, y, \lambda)=0$.

\begin{tabular}{|c|c|c|c|c|}
\hline \multirow[b]{2}{*}{ Degree $n$} & \multicolumn{2}{|c|}{$y=2, \lambda=1 / 2$} & \multicolumn{2}{|c|}{$y=-2, \lambda=1 / 2$} \\
\hline & Real Roots & Complex Roots & Real Roots & Complex Roots \\
\hline 1 & 1 & 0 & 1 & 0 \\
\hline 2 & 0 & 2 & 2 & 0 \\
\hline 3 & 1 & 2 & 3 & 0 \\
\hline 4 & 0 & 4 & 4 & 0 \\
\hline 5 & 1 & 4 & 5 & 0 \\
\hline 6 & 0 & 6 & 4 & 2 \\
\hline 7 & 1 & 6 & 5 & 2 \\
\hline 8 & 0 & 8 & 6 & 2 \\
\hline 9 & 1 & 8 & 7 & 2 \\
\hline 10 & 0 & 10 & 6 & 4 \\
\hline
\end{tabular}

Table 2. Approximate roots of $\mathbf{H}_{n}(x, y, \lambda)=0, x \in \mathbb{R}$.

\begin{tabular}{|c|c|}
\hline Degree $n$ & $x$ \\
\hline 1 & 0 \\
\hline 2 & $-1.7656, \quad 2.2656$ \\
\hline 3 & $-2.7231, \quad 0, \quad 4.2231$ \\
\hline 4 & $-3.2312, \quad-1.4638, \quad 1.6900, \quad 6.0051$ \\
\hline 5 & $-3.2515, \quad-2.7036, \quad 0, \quad 3.2853, \quad 7.6698$ \\
\hline 6 & $-1.2884, \quad 1.4301, \quad 4.8062, \quad 9.2488$ \\
\hline 7 & $-2.3250, \quad 0, \quad 2.8218, \quad 6.2681, \quad 10.762$ \\
\hline 8 & $-2.9891, \quad-1.1791, \quad 1.2772, \quad 4.1775, \quad 7.6817, \quad 12.221$ \\
\hline
\end{tabular}

We can see a regular pattern of the complex roots of the two-variable partially degenerate Hermite equations $\mathbf{H}_{n}(x, y, \lambda)=0$ and also hope to verify a regular pattern of the complex roots of the two-variable partially degenerate Hermite equations $\mathbf{H}_{n}(x, y, \lambda)=0$ (Table 1 ).

We show pattern of real roots of the 2-variable partially degenerate Hermite equations $\mathbf{H}_{n}(x, y, \lambda)=0$ in Figure 4 when $1 \leq n \leq 50, \lambda=1 / 2$.
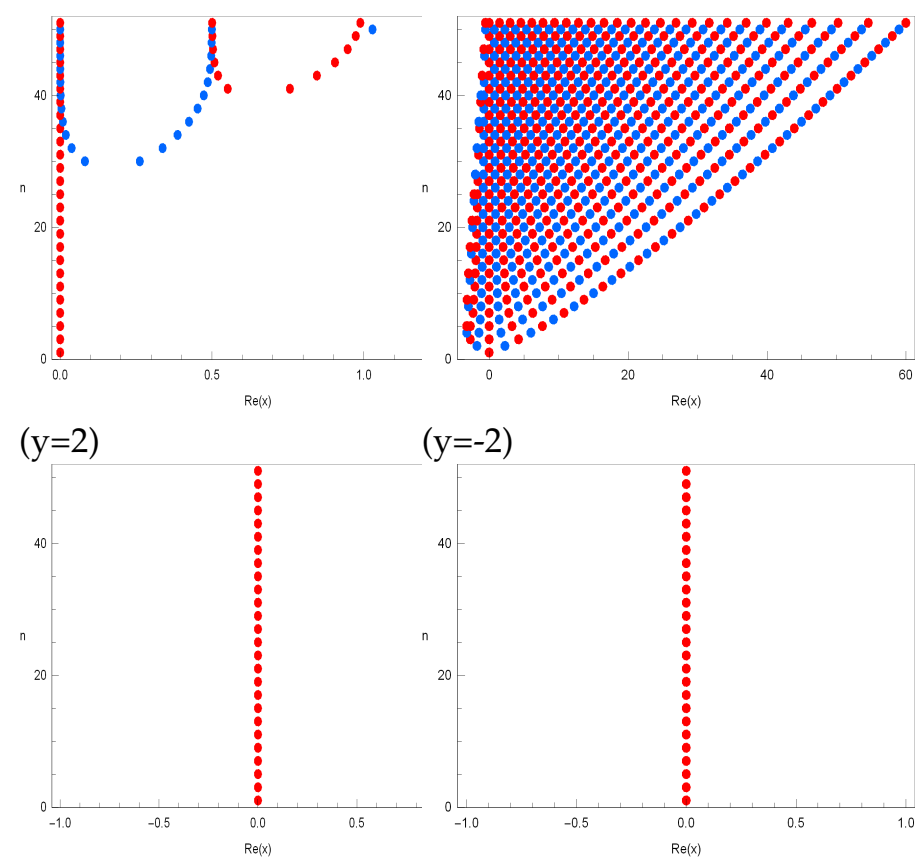

$$
(y=-2+i) \quad(y=-2-i)
$$

Figure 4. Real roots of $\mathbf{H}_{n}(x, y, \lambda)=0$ for $1 \leq n \leq 50, \lambda=\frac{1}{2}$. 
For the top left picture in Figure 4 , we select $y=2$. For the top right picture in Figure 4, we select $y=-2$. For the bottom left picture in Figure 4 , we select $y=-2+i$. For the bottom right picture in Figure 4, we select $y=-2-i$.

Next, we show the approximate roots satisfying $\mathbf{H}_{n}(x, y, \lambda)=0, x \in \mathbb{C}$ for given $n, y=-2$, $\lambda=-1 / 2$ in the Table 2 .

\section{Conclusions and Future Research}

In this article, we made the two-variable partially degenerate Hermite polynomials and get new symmetric identities for those polynomials. We made differential equations induced from the two-variable partially degenerate Hermite polynomials $\mathbf{H}_{n}(x, y, \lambda)$. We also studied the symmetry of the roots of the two-variable partially degenerate Hermite equations $\mathbf{H}_{n}(x, y, \lambda)=0$ for variables $n, y$, and $\lambda$. We show regular patterns of the distribution of roots of equations $\mathbf{H}_{n}(x, y, \lambda)=0$. Therefore, we make several conjectures with numerical calculation:

We use some notations. $R_{\mathbf{H}_{n}(x, y, \lambda)}$ denotes the number of real roots of $\mathbf{H}_{n}(x, y, \lambda)=0$ on the real plane, that is, $\operatorname{Im}(x)=0$ and $C_{\mathbf{H}_{n}(x, y, \lambda)}$ denotes the number of complex roots of $\mathbf{H}_{n}(x, y, \lambda)=0$, where $n$ is the degree of the polynomial $\mathbf{H}_{n}(x, y, \lambda)$. Then, we have $R_{\mathbf{H}_{n}(x, y, \lambda)}=n-C_{\mathbf{H}_{n}(x, y, \lambda)}$. We see that the complex roots of equations $\mathbf{H}_{n}(x, y, \lambda)=0$ for given $y$ and $\lambda$ have a regular pattern. Therefore, we make the below conjecture.

Conjecture 1. For odd positive integer $n$. If $a>0$ or $a \in \mathbb{C} \backslash\{a \mid a<0\}$, prove or disprove that

$$
R_{\mathbf{H}_{n}(x, a, \lambda)}=1, \quad C_{\mathbf{H}_{n}(x, a, \lambda)}=2\left[\frac{n}{2}\right],
$$

where $\mathbb{C}$ is the set of complex numbers.

Conjecture 2. For odd positive integer $n$ and $a \in \mathbb{C}$, prove or disprove that

$$
\mathbf{H}_{n}(0, a, \lambda)=0 \text {. }
$$

It is still unknown if Conjecture 1 and Conjecture 2 are true or not for all variables $y$ and $\lambda$.

Conjecture 3. Prove that the roots of $\mathbf{H}_{n}(x, a, \lambda)=0, a \in \mathbb{R}$, are symmetrical about $\operatorname{Im}(x)=0$ for all $a \in \mathbb{R}$. Prove that the roots of $\mathbf{H}_{n}(x, a, \lambda)=0$ are not symmetrical about $\operatorname{Im}(x)=0$ for all $a<0, a \in \mathbb{C} \backslash \mathbb{R}$.

Finally, we would like to know how many roots $\mathbf{H}_{n}(x, y, \lambda)=0$ has. We would like to know $C_{\mathbf{H}_{n}(x, y, \lambda)}$ of $\mathbf{H}_{n}(x, y, \lambda)=0$.

Conjecture 4. For $a \in \mathbb{C}$, prove or disprove that $\mathbf{H}_{n}(x, a, \lambda)=0$ has $n$ distinct solutions.

Our new approach using the numerical method about the roots of equations $\mathbf{H}_{n}(x, y, \lambda)=0$ is one of the directions to know new information.

Author Contributions: We are all equally contributed to write this paper. All authors have read and agreed to the published version of the manuscript.

Funding: This work was supported by the Dong-A University research fund.

Conflicts of Interest: The authors declare no conflict of interest.

\section{References}

1. Andrews, L.C. Special Functions for Engineers and Mathematicians; Macmillan. Co.: New York, NY, USA, 1985.

2. Appell, P.; Hermitt Kampé de Fériet, J. Fonctions Hypergéomtriques et Hypersphériques: Polynomes d Hermite; Gauthier-Villars: Paris, France, 1926. 
3. Erdelyi, A.; Magnus, W.; Oberhettinger, F.; Tricomi, F.G. Higher Transcendental Functions; Krieger: New York, NY, USA, 1981; Volume 3.

4. Andrews, G.E.; Askey, R.; Roy, R. Special Functions; Cambridge University Press: Cambridge, UK, 1999.

5. Arfken, G. Mathematical Methods for Physicists, 3rd ed.; Academic Press: Orlando, FL, USA, 1985.

6. Carlitz, L. Degenerate Stiling, Bernoulli and Eulerian numbers. Util. Math. 1979, 15, 51-88.

7. Young, P.T. Degenerate Bernoulli polynomials, generalized factorial sums, and their applications. J. Number Theorey 2008, 128, 738-758. [CrossRef]

8. Ryoo, C.S. Notes on degenerate tangent polynomials. Glob. J. Pure Appl. Math. 2015, 11, 3631-3637.

9. Haroon, H.; Khan, W.A. Degenerate Bernoulli numbers and polynomials associated with degenerate Hermite polynomials. Commun. Korean Math. Soc. 2018, 33, 651-669.

10. Kim, T.; Kim, D.S. Identities involving degenerate Euler numbers and polynomials arising from non-linear differential equations. J. Nonlinear Sci. Appl. 2016, 9, 2086-2098. [CrossRef]

11. Hwang, K.W.; Ryoo, C.S. Differential equations associated with two variable degenerate Hermite polynomials. Mathematics 2020, 8, 228. [CrossRef]

12. Hwang, K.W.; Ryoo, C.S.; Jung, N.S. Differential equations arising from The generating function of The $(r, \beta)$-Bell Polynomials and distribution of zeros of equations. Mathematics 2019, 7, 736. [CrossRef]

13. Ryoo, C.S. A numerical investigation on The structure of The zeros of The degenerate Euler-tangent mixed-type polynomials. J. Nonlinear Sci. Appl. 2017, 10, 4474-4484. [CrossRef]

14. Ryoo, C.S. Differential equations associated with tangent numbers. J. Appl. Math. Inform. 2016, 34, 487-494. [CrossRef]

15. Ryoo, C.S. Some identities involving Hermitt Kampé de Fériet polynomials arising from differential equations and location of their zeros. Mathematics 2019, 7, 23. [CrossRef]

16. Ryoo, C.S.; Agarwal, R.P.; Kang, J.Y. Differential equations associated with Bell-Carlitz polynomials and their zeros. Neural Parallel Sci. Comput. 2016, 24, 453-462.

17. Yang, S.L.; Qiao, Z.K. Some symmetry identities for The Euler polynomials. J. Math. Res. Expos. 2010, $30,457-464$.

(C) 2020 by the authors. Licensee MDPI, Basel, Switzerland. This article is an open access article distributed under the terms and conditions of the Creative Commons Attribution (CC BY) license (http://creativecommons.org/licenses/by/4.0/). 